\title{
Intervening in Earth's climate system through space-based solar reflectors
}

\author{
F.J.T. Salazar ${ }^{a, *}$, C.R. McInnes ${ }^{\mathrm{a}}$, O.C. Winter $^{\mathrm{b}}$ \\ ${ }^{a}$ School of Engineering, University of Glasgow, Glasgow G12 8QQ, United Kingdom \\ ${ }^{\mathrm{b}}$ UNESP-Grupo de Dinâmica Orbital e Planetologia, Guaratinguetá, SP 12516-410, Brazil
}

Received 20 December 2015; received in revised form 5 April 2016; accepted 6 April 2016 Available online 12 April 2016

\begin{abstract}
Several space-based climate engineering methods, including shading the Earth with a particle ring for active cooling, or the use of orbital reflectors to increase the total insolation of Mars for climate warming have been considered to modify planetary climates in a controller manner. In this study, solar reflectors on polar orbits are proposed to intervene in the Earth's climate system, involving near circular polar orbits normal to the ecliptic plane of the Earth. Similarly, a family of displaced polar orbits (non-Keplerian orbits) are also characterized to mitigate future natural climate variability, producing a modest global temperature increase, again to compensate for possible future cooling. These include deposition of aerosols in the stratosphere from large volcanic events. The two-body problem is considered, taking into account the effects of solar radiation pressure and the Earth's $J_{2}$ oblateness perturbation.
\end{abstract}

(C) 2016 COSPAR. Published by Elsevier Ltd. All rights reserved.

Keywords: Space reflectors; Polar orbits; Non-Keplerian orbits; Earth's climate system; $J_{2}$ perturbation; Two-body problem

\section{Introduction}

Simple climate models help to explain the natural variability of the Earth's climate system (McGuffie and Henderson-Sellers, 2005). These models show that Earth's climate can switch from a stable warm state to a cool state and is sensitive to relative small changes in solar insolation (Berglund and Gentz, 2001; Emanuel, 2002; Allen et al., 2006). The periodicity of ice ages (Milankovitch cycles) (Muller and MacDonald, 1997) can be explained by these processes. These cycles are due to a combination between oscillations in the elements of the Earth's orbit about the Sun, and periodic changes to the orientation of the Earth's spin axis, which change the relative flux of energy received

\footnotetext{
* Corresponding author. Tel.: +55 12981015572.

E-mail addresses: e7940@hotmail.com (F.J.T. Salazar), Colin.McInnes@ glasgow.ac.uk (C.R. McInnes), ocwinter@gmail.com (O.C. Winter).
}

by the Earth at polar and equatorial latitudes. Although the total change in insolation due to Milankovitch cycles is less than $1 \%$, the distribution of heat input as a function of latitude seems to be the main effect (e.g. insolation at high latitudes directly effects the growth and retreat of ice sheets). Similarly, while human-driven climate warming is of contemporary concern, volcanic aerosols reflect sunlight to space and thus reduce solar heating of the Earth, therefore large volcanic-driven forcing can have a significant short-term cooling effect (Angell, 1988; Angell and Korshover, 1984; Zuev et al., 2015), such as Tambora in 1815 (Hansen et al., 1992) or catastrophic super-volcano events, e.g. Toba mega-eruption approx. 71,000 years ago (Zielinski et al., 1996). However, if a period similar to the 'little ice age' (1645-1715) recurred (Le Roy Ladurie, 1971; Free and Robock, 1999), or indeed future large volcanic events occurred, then there could be significant economic consequences for energy demand and agriculture (i.e. energy prices and economic stability). Therefore, it is 
interesting to consider active strategies to avoid such shortterm climate change.

Space-based geo-engineering proposals aim to intervene in the climate system by deliberately modifying the Earth's energy balance to reduce or increase the global mean temperature in a controlled manner, with presumed beneficial effect (McInnes, 2010). For example, space-based solar shields have been proposed by various authors to decrease the total solar insolation. Hudson (1991) proposed the deployment of a $10^{11} \mathrm{~kg}$ 'space parasol' at the $L_{1}$ Langrangian point of the Earth-Sun system to intercept some desired fraction of the solar radiant energy. Similarly, McInnes (2002) proposed the use of a $4 \times 10^{11} \mathrm{~kg}$ metallic reflector located sunward of the Sun-Earth interior Lagrange point to offset increases in mean global surface temperature. Angel (2006) also considered cooling the Earth with a cloud of small spacecraft orbited near the inner Lagrange point. In Earth-orbit-based systems, Pearson et al. (2006) proposed an artificial planetary ring about the Earth, composed of passive scattering particles, delivered from the Earth, Moon, or asteroids, and attitude-controlled spacecraft with parasols. However, since thin film devices require terrestrial fabrication and launch at extremely high cost, it is possible that much simpler partly reflecting disks could be extracted from captured near Earth asteroids (McInnes, 2006; Sanchez and McInnes, 2011). Recently, Bewick et al. (2012, 2013) proposed a scheme for dust cloud and heliotropic rings to reduce the manufacturing requirement for space-based geoengineering at the Earth-Sun $L_{1}$ Lagrangian point and medium Earth orbits, respectively. The use of mass drivers to eject material from the asteroid surface, in such a way that the asteroid would be stabilized near $L_{1}$, would still be more efficient than lifting material from the surface of the Earth.

In the last forty years, similar geo-engineering schemes have been the subject of numerous studies for a possible futuristic use of orbiting solar reflectors for illuminationfrom-space applications, e.g. providing extra hours of illumination for energy supplies or terraforming schemes (engineering an Earth-like climate) (Glaser, 1968; Ehricke, 1979; Oberg, 1981; Canady and Allen, 1982; Fogg, 1995). However, as early as 1929, Oberth (1972) had already proposed the use of 'space mirrors' for solar power generation on Earth. The main advantage is the vast energy leverage delivered by the reflectors which is obtained in a relatively short time (Maunter and Parks, 1990). Modest-sized reflectors, of about $20-25 \mathrm{~m}$ in diameter, have already flown in space, such as the Russian Znamya space mirror experiment (Leary, 1993). Although the first space mirror experiment (Znamya 2) was a successful, the spot brightness achievable with reflectors of this size is a tiny fraction of the mid-day Sun. For example, McInnes (2002) showed that the required reflector area to increase the total insolation of Mars by $30 \%$, as part of a largescale terraforming effort, is of order $10^{13} \mathrm{~m}^{2}$ (and mass of order $10^{10} \mathrm{~kg}$ ). Similarly, Bewick et al. (2011) proposed the use of a set of 300 Sun-pointing orbiting reflectors with a total system mass of 370 tonnes, to provide sufficient illumination onto the lunar surface to enable the survivability of missions in the long periods of the lunar night. In this manner, large-scale geo-engineering appears to be an interesting tool to explore the possibility of climate heating in order to manage fast cooling events that have occurred in the distant past.

The work presented in this paper aims to investigate the feasibility of using orbiting reflectors on polar orbits in order to explore the possibility of an increase of the total planetary insolation of $0.5 \%$ (equivalent to increasing the mean global temperature by $0.5 \mathrm{~K}$ ) to mitigate against possible large scale climate cooling (Teller et al., 2004). As suggested in McInnes (2010), two candidate orbits for solar reflectors will be evaluated: a Sun-synchronous frozen polar orbit normal to the ecliptic plane of Earth and displaced, non-Keplerian circular orbits. In principle, reflector orbits normal to the Sun-line are more efficient than orbits in the ecliptic plane. This fixed orientation provides additional solar energy transferred to the surface of the Earth. However, reflectors deployed directly in Keplerian orbits about the Earth are strongly perturbed by solar radiation pressure. In this case, displaced circular orbits are an interesting alternative to manage the momentum accumulated by the reflectors. They are essentially circular, near polar orbits but, due to the effect of solar radiation pressure, the orbits are displaced behind Earth along the anti-Sun line (McInnes and Simmons, 1992). In this light, the twobody problem is considered for polar and displaced orbits, including solar radiation pressure (SRP) and the effect of the Earth's oblateness, the $J_{2}$ effect. It will be shown that SRP and solar reflector orientation has a significant effect on the orbital evolution. Additionally, the $J_{2}$ term will be considered to obtain analytical expressions for the required pitch angle and characteristic acceleration of nonKeplerian equilibrium solutions in a rotating frame of reference. The linear stability of the orbit families will be also investigated. Finally, using the reflector mean distance from the Earth, the area-to-mass ratio and the angle of incidence, the required total reflector area for a $0.5 \%$ increase in total insolation will be found.

The remainder of the paper is organized as follows. Section 2 describes a zero-dimensional energy balance model (EBM) and the basic concept for illumination from space. Section 3 describes the necessary conditions to achieve Sun-synchronous frozen orbits considering SRP and the $J_{2}$ effect, and numerical experiments for different values of reflector orientation and characteristic acceleration. Section 4 determines the existence and stability of a family of displaced circular orbits when viewed from an internal frame of reference. Section 5 discusses the mass required to fabricate the solar reflector utilizing the polar orbits found in the previous sections. Finally, the conclusions together with the discussion are drawn in Section 6. 


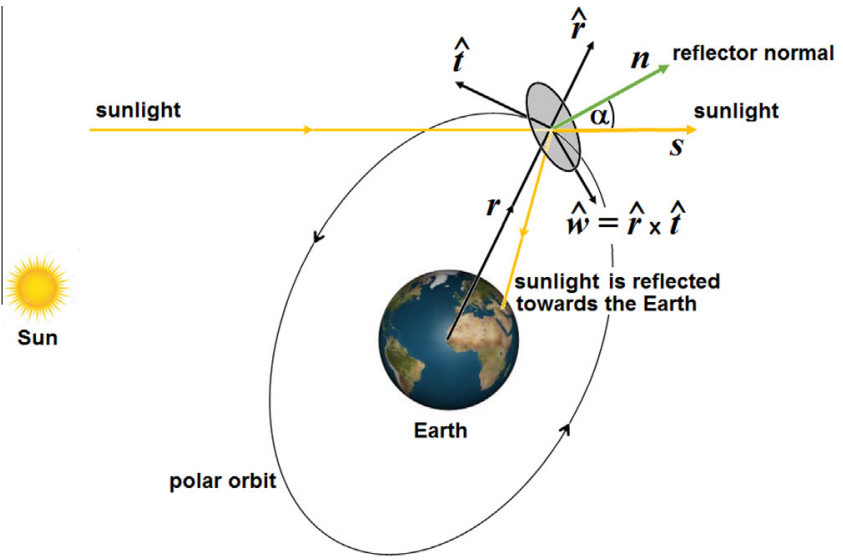

(a) solar radiation force

$$
T=\kappa \cos ^{2} \alpha n
$$

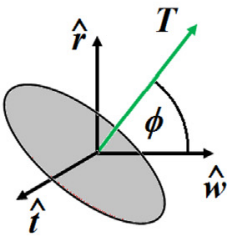

(b)

Fig. 1. (a) Solar reflector on a polar orbit and radial, transverse and normal (RTN) frame, (b) Solar radiation force.

\section{Climate engineering}

In order to investigate the response of the Earth's climate to large-scale engineering interventions, it is necessary to determine a relation between global mean temperature and solar insolation, in such a way that the engineering requirements for the fabrication of the space-based solar reflectors can be obtained.

\subsection{Energy balance model (EBM)}

The simplest method of considering the climate system of the Earth is in terms of its global energy balance (McGuffie and Henderson-Sellers, 2005). Based on the principle of conservation of energy, the EBM represents an accounting of the balance between the total incoming radiation received from the Sun and outgoing radiation, i.e., radiation reflected and emitted from the Earth, including the atmosphere.

The lowest order model is the zero dimensional model, which considers the Earth as a single point in space having a global mean effective temperature $\bar{T}$. Although it does not have the potential of numerical General Circulation Models (GCM), the zero dimensional model provides globally averaged estimates of the climate responses. From this perspective, the total energy received from the Sun per unit time is $4 \pi R^{2} I$, where $R=6371 \mathrm{~km}$ is the radius of the Earth and $I=342.5 \mathrm{~W} \mathrm{~m}^{-2}$ is the total solar insolation. ${ }^{1}$ Similarly, the reflected solar radiation per unit time is $4 \pi R^{2} I \alpha_{E}$ and the emitted infrared radiation per unit time is $4 \pi R^{2} \epsilon \sigma \bar{T}^{4}$, where $\alpha_{E} \approx 0.3$ is the mean planetary albedo, $\epsilon \approx 0.62$ is the mean emissivity and $\sigma=5.67 \times 10^{-8} \mathrm{~W} \mathrm{~m}^{-2}$ $\mathrm{K}^{-4}$ is the Stefan-Bolztman constant. Hence, the mean temperature can be estimated by considering the radiation balance,

\footnotetext{
${ }^{1}$ The solar insolation is defined in terms of solar flux $F=1370 \mathrm{~W} \mathrm{~m}^{-2}$ as $I=F / 4$.
}

$4 \pi R^{2} I=4 \pi R^{2} I \alpha_{E}+4 \pi R^{2} \epsilon \sigma \bar{T}^{4}$.

In this case, an initial estimate of the mean Earth's temperature can be obtained from Eq. (1) as

$\bar{T}=\left(\frac{I\left(1-\alpha_{E}\right)}{\epsilon \sigma}\right)^{1 / 4}$,

which yields an estimated mean temperature of $14^{\circ}$, a value approximately similar with the observed annual average temperature for the Earth.

\subsection{Reflection of light onto a planetary surface}

The basic concept for illumination from space is shown in Fig. 1(a). In this figure, the solar reflectors are in a polar orbit. The Sun's rays are emanating from the left. Note that the solar reflector orientation is defined by the unit vector $\boldsymbol{n}$ normal to the reflector surface. In addition, the angle of incidence is defined by the reflector pitch angle $\alpha$, i.e., the angle between the reflector normal and the unit vector $s$ of the incoming sunlight.

If we refer again to Fig. 1(a) and assume only the loss related to the angle of incidence, the total energy reflected by the mirror is $F A \cos \alpha$, where $F$ is the solar flux and $A$ is the total reflective area. Similarly, the total energy reflected onto the Earth's surface is $4 \pi R^{2} \delta I$, where $\delta I$ is the radiant intensity on the Earth. Since the energy reflected from the mirror is equal to the energy radiated on the Earth, then the increase in insolation can be estimated as

$\delta I=\frac{F A}{4 \pi R^{2}} \cos \alpha$.

The new surface temperature can therefore be written as

$\bar{T}=\left(\frac{I\left(1-\alpha_{E}\right)+\delta I}{\varepsilon \sigma}\right)^{1 / 4}$.

Now that the single energy balance model has been described, family of orbits to manipulate the solar insolation will be considered. 


\section{Sun-synchronous orbit}

A Sun-synchronous orbit is a geocentric orbit which combines the altitude of the satellite and the inclination of the orbit, in such a way that, an object on that orbit will ascend or descend over any given point of the Earth at the same local mean solar time (Tscherbakova et al., 1999). This orbit is achieved when the speed of precession of the osculating orbital plane is approximately one degree per day with respect to the celestial sphere to keep pace with the Earth's revolution around the Sun. A frozen orbit is an orbit where the mean argument of the perigee, the eccentricity and the inclination remain constant (Coffey et al., 1994). In this light, Sun-synchronous frozen orbits are one of the most frequently used orbits for Earth science missions (e.g. remote sensing). The typical Sunsynchronous orbit tends to be near-polar and slightly retrograde. Thus, a solar reflector could be deployed directly in a Sun-synchronous frozen orbit and its orientation could also be fixed as the reflector orbits normal to the Sunline, providing an additional benefit over orbits in the ecliptic plane (McInnes, 2010).

\subsection{Orbital dynamics}

In the two-body problem, the plane of any orbit will remain fixed with respect to an inertial frame as the Earth rotates beneath it, i.e., the orbital elements do not change with time in the inertial reference coordinates. However, the non-spherical gravitational potential perturbation, as well as the solar radiation pressure force (SRPF), could cause this plane to slowly shift. Thus, in this study $J_{2}$ and solar pressure perturbations are considered to model the dynamics of the solar reflector. The variations of the orbital elements are governed by the Gauss' form of the planetary equations. The dynamical equation of motion of the solar reflector can be written as (Battin, 1987)

$$
\begin{aligned}
\frac{\mathrm{d} a}{\mathrm{~d} t}= & \frac{2 e \sin f}{n \sqrt{1-e^{2}}} p_{r}+\frac{2 a \sqrt{1-e^{2}}}{n r} p_{t}, \\
\frac{\mathrm{d} e}{\mathrm{~d} t}= & \frac{\sqrt{1-e^{2}} \sin f}{n a} p_{r}+\frac{\sqrt{1-e^{2}}}{n a^{2} e}\left(\frac{a p}{r}-r\right) p_{t}, \\
\frac{\mathrm{d} i}{\mathrm{~d} t}= & \frac{r \cos (f+\omega)}{n a^{2} \sqrt{1-e^{2}}} p_{w}, \\
\frac{\mathrm{d} \Omega}{\mathrm{d} t}= & \frac{r \sin (f+\omega)}{n a^{2} \sqrt{1-e^{2}} \sin i} p_{w}, \\
\frac{\mathrm{d} \omega}{\mathrm{d} t}= & -\frac{\sqrt{1-e^{2}} \cos f}{n a e} p_{r}+\frac{p}{e h}\left[\sin \theta\left(1+\frac{1}{1+e \cos f}\right)\right] p_{t} \\
& -\frac{r \cot i \sin (f+\omega)}{n a^{2} \sqrt{1-e^{2}}} p_{w}, \\
\frac{\mathrm{d} f}{\mathrm{~d} t}= & \frac{h}{r^{2}}+\frac{1}{e h}\left[\frac{h^{2}}{\mu} \cos f p_{r}-\left(r+\frac{h^{2}}{\mu}\right) \sin f p_{t}\right],
\end{aligned}
$$

where $\Omega$ is the right ascension of the ascending node (or simply 'node'), $\omega$ is the argument of perigee, $f$ is the true anomaly, $r=a\left(1-e^{2}\right) /(1+e \cos f)$ is the distance from the Earth, $p=a\left(1-e^{2}\right)$ is the semi-latus rectum, $n=\sqrt{\mu / a}$ is the mean motion, $h=\sqrt{\mu p}$ is the angular momentum, and $p_{r}, p_{t}, p_{w}$ are the acceleration components of the perturbation force $\boldsymbol{p}$ along radial $\hat{\boldsymbol{r}}$, transverse $\hat{\boldsymbol{t}}$ and normal $\hat{\boldsymbol{w}}=\hat{\boldsymbol{r}} \times \hat{\boldsymbol{t}}$ directions (RTW frame), respectively, as shown in Fig. 1(a). The perturbing acceleration $\boldsymbol{p}$ is expressed analytically as follows (Battin, 1987):

$$
\begin{aligned}
& p_{r}=-\frac{3}{2} \frac{J_{2} \mu R^{2}}{r^{4}}\left[1-3 \sin ^{2} i \sin ^{2}(\omega+f)\right]+T_{r}, \\
& p_{t}=-\frac{3}{2} \frac{J_{2} \mu R^{2}}{r^{4}} \sin ^{2} i \sin 2(\omega+f)+T_{t}, \\
& p_{w}=-\frac{3}{2} \frac{J_{2} \mu R^{2}}{r^{4}} \sin 2 i \sin (\omega+f)+T_{w},
\end{aligned}
$$

where $J_{2}=0.00108263$ is a dimensionless constant corresponding to oblateness of the Earth, $\mu=398600.440 \mathrm{~km}^{3}$ $\mathrm{s}^{-2}$ is the gravitational parameter of the Earth (Bate et al., 1971), and $T_{r}, T_{t}, T_{w}$ are the radial, transverse and normal acceleration components, respectively, of the solar radiation force $\boldsymbol{T}$ as shown in Fig. 1(b).

The classical orbit elements are defined in the J200 Earth-Centered Internial (ECI) frame, where $X$-axis points to the J2000 equinox, $Y$-axis lies in the Earth's equatorial plane orthogonally to the $X$-axis, and $Z$-axis is directed to the Earth's north pole.

Work presented by Gong et al. (2012) on orbital dynamics using solar sails obtains analytical expressions for the SRPF components, such that, the conditions of a Sunsynchronous frozen orbit are satisfied. Firstly, setting the transverse component equal to zero (i.e. $T_{t}=0$ ), the semi-major axis and the eccentricity remain constant over one orbital period (Gong et al., 2012). Therefore, the normal of the reflector lies in the plane spanned by the position vector and $Z$-axis. In addition, the variation of the inclination is equal to zero over one orbital period when the argument of perigee is a multiple of $\pi / 2$ (i.e. $\omega= \pm k \pi / 2$ ) (Gong et al., 2012). Therefore, a Sun-synchronous frozen orbit is finally achieved when the argument of perigee always remains constant over one orbital period and the node keeps pace with the Sun-line. According to Gong et al. (2012), the radial and normal components of the SRPF that satisfy the Sun-synchronous conditions can be obtained as

$$
\begin{aligned}
& T_{r}=\sqrt{\frac{\mu}{a\left(1-e^{2}\right)}} \cos i n_{\odot}+\frac{3 \mu J_{2} R^{2}}{4 a^{4}\left(1-e^{2}\right)^{5 / 2}}\left(1-e \cos ^{2} i\right), \\
& T_{w}=-\frac{\mu J_{2} R^{2} \sin 2 i}{2\left(1-e^{2}\right)^{3 / 2} a^{4} e \sin \omega}-\frac{2}{3} \sqrt{\frac{\mu\left(1-e^{2}\right)}{a e^{2}}} \frac{\sin i n_{\odot}}{\sin \omega},
\end{aligned}
$$

where $n_{\odot}=0.9856 \mathrm{deg} /$ day is the mean motion of the Sun. Note that Eq. (7) is singular for circular orbits.

Denoting by $\phi$ the angle between the reflector normal and the normal component in the RTN frame (see Fig. 1 (b)), then the SRPF in the RTN frame is given by 
$T_{r}=T \sin \phi$,

$T_{t}=0$,

$T_{w}=T \cos \phi$,

where $T$ is the magnitude of the SRPF. Thus,

$\phi=\tan ^{-1} \frac{T_{r}}{T_{w}}$.

If the angle between the reflector normal and the radial vector remains constant, then Eqs. (7)-(9) permit the semimajor axis of a Sun-synchronous frozen orbit, as well as the solar radiation force, to be obtained as a function of the classical orbit elements for a given angle $\phi$ :

$a=\left(-\frac{\frac{3 \mu J_{2} R^{2}}{4\left(1-e^{2}\right)^{5 / 2}}\left(1-e \cos ^{2} i\right)+\frac{\mu J_{2} R^{2} \sin 2 i}{2\left(1-e^{2}\right)^{3 / 2} e \sin \omega} \tan \phi}{\sqrt{\frac{\mu}{1-e^{2}}} \cos i n_{\odot}+\frac{2}{3} \sqrt{\frac{\mu\left(1-e^{2}\right)}{e^{2}}} \frac{\sin i n_{\odot}}{\sin \omega} \tan \phi}\right)^{2 / 7}$,

$T=\sqrt{1+T_{w}^{2}} \tan \phi$.

In this manner, Fig. 2 shows the semi-major axis and the magnitude of SRPF as a function of inclination for a nearcircular orbit (i.e. $e=0.05$ ) and different given values of $\phi$. The argument of perigee was chosen following the Sun-synchronous frozen condition $\omega=-\pi / 2$.

Fig. 2(d) suggests that SRPF is minimum when the inclination of the frozen orbit is about $112^{\circ}$ and the semi-major axis is about $9362 \mathrm{~km}$, regardless of the angle $\phi$. This inclination is approximately equal to the inclination of the plane perpendicular to the Earth's ecliptic plane, which is inclined to Earth's equatorial plane by an angle $\varepsilon$ of about $23.4^{\circ}$. In addition, no significant variation of semimajor axis and inclination with regards to the eccentricity, such that the SRPF is minimum, was found. Therefore, Fig. 2 identifies an initial condition for which the variation of classical orbit elements is minimized. The Keplerian elements identified through Eq. (10) are now used as initial conditions for a numerical propagation of Eq. (5), forward in time, over a period of 5 years. The numerical integration of Eq. (5) is performed through the Bulirsch-Stoer algorithm (Stoer and Bulirsch, 1980; Press et al., 1992), programmed in the $\mathrm{C}$ language, choosing a dimensionless step size $h=1.0 \times 10^{-3}$ and setting a local truncation error of $1.0 \times 10^{-9}$.

Once the initial parameters of the orbital dynamics are found, the solar acceleration components $T_{r}, T_{t}$, and $T_{w}$ in Eq. (6) to perform the numerical propagation are determined by

$T_{r}=\kappa \cos ^{2} \alpha \cos \phi$,

$T_{t}=0$,

$T_{w}=\kappa \cos ^{2} \alpha \sin \phi$,

where $\kappa$ is the characteristic acceleration of the reflector (McInnes, 2004) and the pitch angle $\alpha$ can be obtained from the relation $\cos \alpha=\boldsymbol{n} \cdot \boldsymbol{s}$ (see Fig. 1(a)). The acceleration induced by the SRPF is defined by $\kappa=2 P \sigma_{A}^{-1}$ where (a)

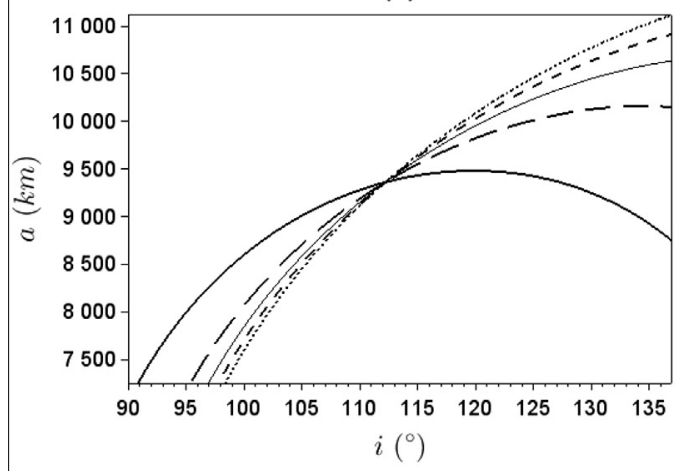

(c)

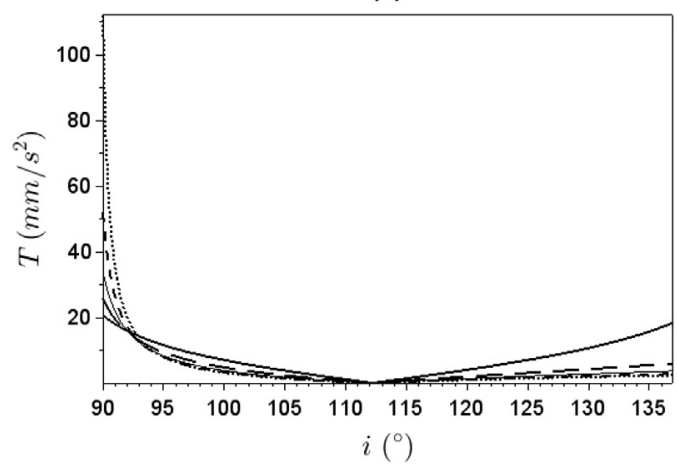

(b)

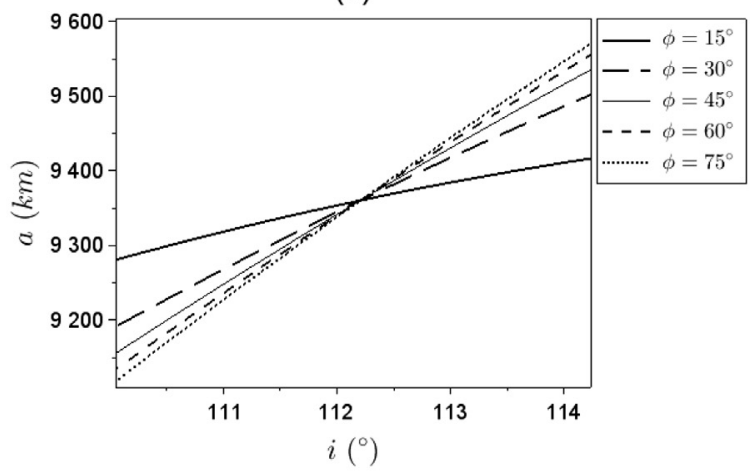

(d)

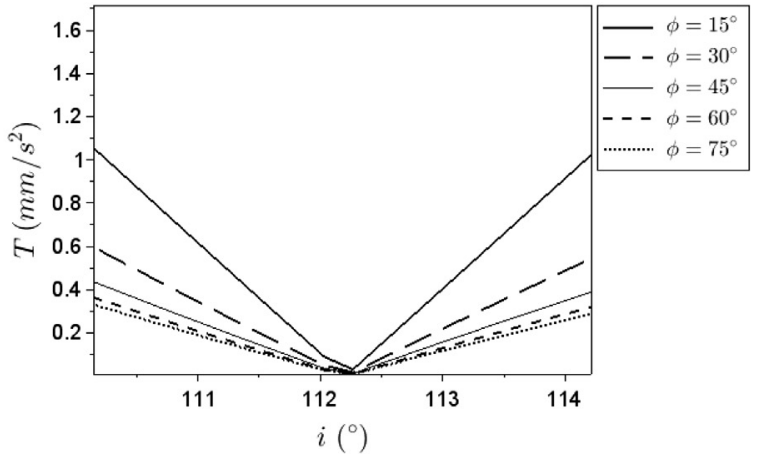

Fig. 2. Semi-major axis $(\mathrm{a}, \mathrm{b})$ and $\operatorname{SRPF}(\mathrm{b}, \mathrm{c})$ as a function of inclination for a near-circular orbit, i.e. $e=0.05$, and different given values of $\phi$. 
$P$ is the solar radiation pressure at the distance of Earth from the Sun $\left(4.46 \times 10^{-6} \mathrm{Nm}^{-2}\right)$ and $\sigma_{A}$ is the mass per unit area of the reflector.

The unit vectors $\boldsymbol{n}$ and $\boldsymbol{s}$ of the reflector normal and the sunlight, respectively, in the RTN frame are given by Gong et al. (2012)

$\boldsymbol{n}=\left[\begin{array}{lll}\sin \phi & 0 & \cos \phi\end{array}\right]^{\mathrm{T}}$,

$s=-R_{3}(\omega+f) R_{1}(i) R_{3}(\Omega)\left[\cos \lambda_{\odot} \cos \varepsilon \sin \lambda_{\odot} \sin \varepsilon \cos \lambda_{\odot}\right]^{\mathrm{T}}$,

where superscript ' $\mathrm{T}$ ' means transpose, $\varepsilon=23.4^{\circ}$ is the mean obliquity of the ecliptic, $\lambda_{\odot}$ is the longitude of Sun measured in the Earth's equatorial plane from $X$-axis, and the transition matrices are given by

$R_{1}(\theta)=\left[\begin{array}{ccc}1 & 0 & 0 \\ 0 & \cos \theta & \sin \theta \\ 0 & -\sin \theta & \cos \theta\end{array}\right], R_{3}(\theta)=\left[\begin{array}{ccc}\cos \theta & \sin \theta & 0 \\ -\sin \theta & \cos \theta & 0 \\ 0 & 0 & 1\end{array}\right]$.

Assuming that the longitude of the Sun is a linear function of time, then

$\lambda_{\odot}=\lambda_{\odot 0}+n_{\odot} t$.

Here, $\lambda_{\odot 0}$ is the initial solar longitude at the instant when the reflector is deployed in the Sun-synchronous frozen orbit. The other four parameters $(\omega, f, i, \Omega)$ in Eq. (13) can be found from the numerical solutions of Eq. (5).
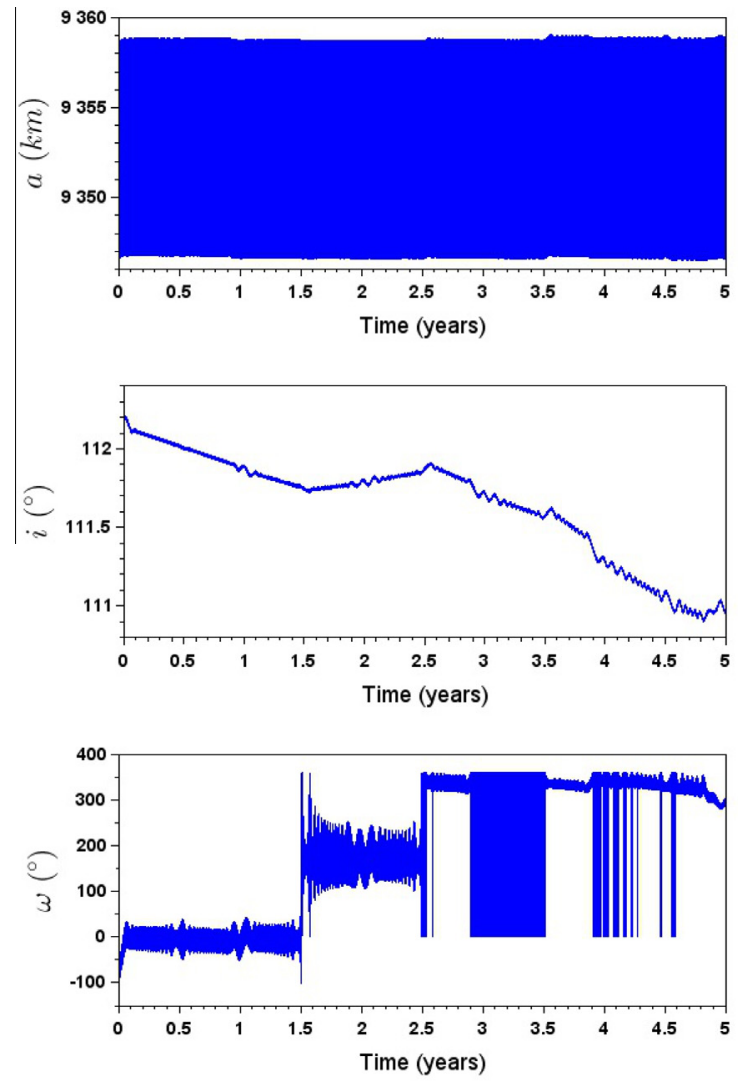

Figs. 3 and 4 show a numerical simulation of the variation of the classical orbit elements of the reflector, as well as the variation of the pitch angle $\alpha$ during 5 years for a given characteristic acceleration, such that, the reflector normal remains constant in the RTN frame. The transverse component of the SRPF is equal to zero to keep the semimajor axis and eccentricity unchanged over each orbit period, and the angle between the reflector normal and the normal component in the RTN frame used for this simulation is $\phi=55^{\circ}$. The simulation parameters are given in Table 1 .

For a constant direction of the SRPF in the RTN frame with transverse component equal to zero, the semi-major axis and the inclination of a near-circular orbit practically remain unchanged for several years as shown in Fig. 3 and Fig. 4. Note that, although the variation of these two orbital elements, including the eccentricity, is small, their longterm evolution is determined by the Earth's motion around the Sun. However, the variation of the argument of perigee leads to a loss of synchronization with the sunlight direction as shown in Fig. 3. Therefore, the reflector normal angle $\phi$ should be adjusted to control the Keplerian elements to follow their desired values and achieve a Sunsynchronous orbit as suggested by Gong et al. (2012). The design of an attitude control for a large solar reflector spacecraft introduces engineering challenges (Hedgepeth et al., 1981). For example, Borggräfe et al. (2014, 2015)
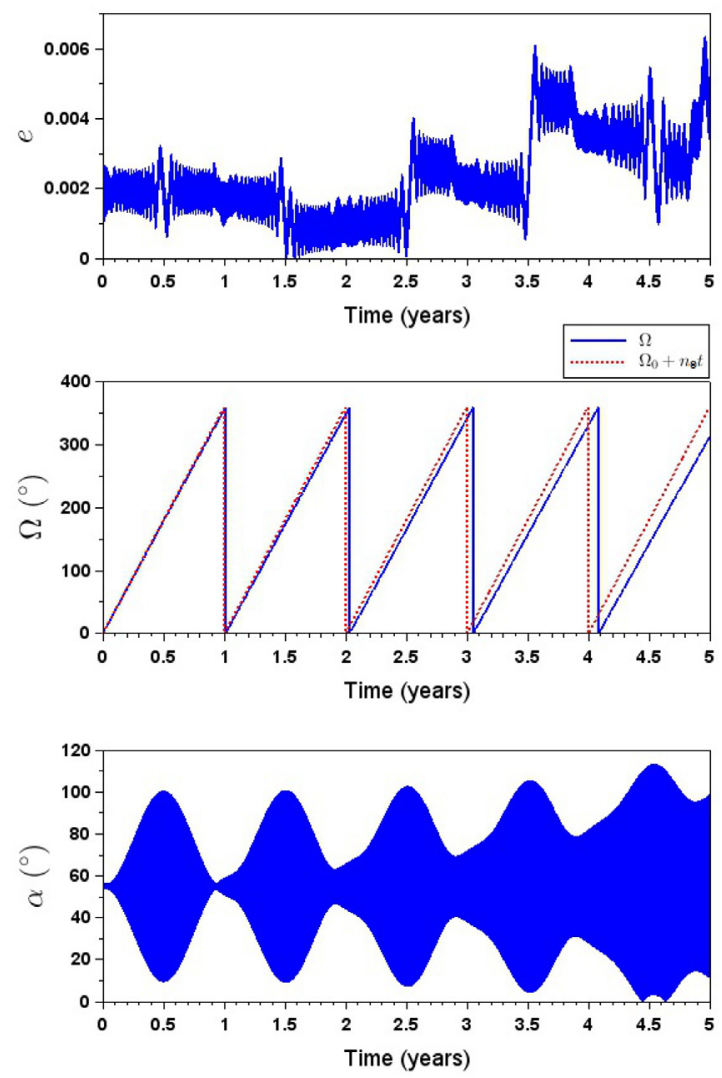

Fig. 3. Classical orbit elements of a Sun-synchronous orbit and Sun angle variation with $\phi=55^{\circ}$ and $\kappa=1.0 \mathrm{~mm} / \mathrm{s}^{2}$ during 5 years. 

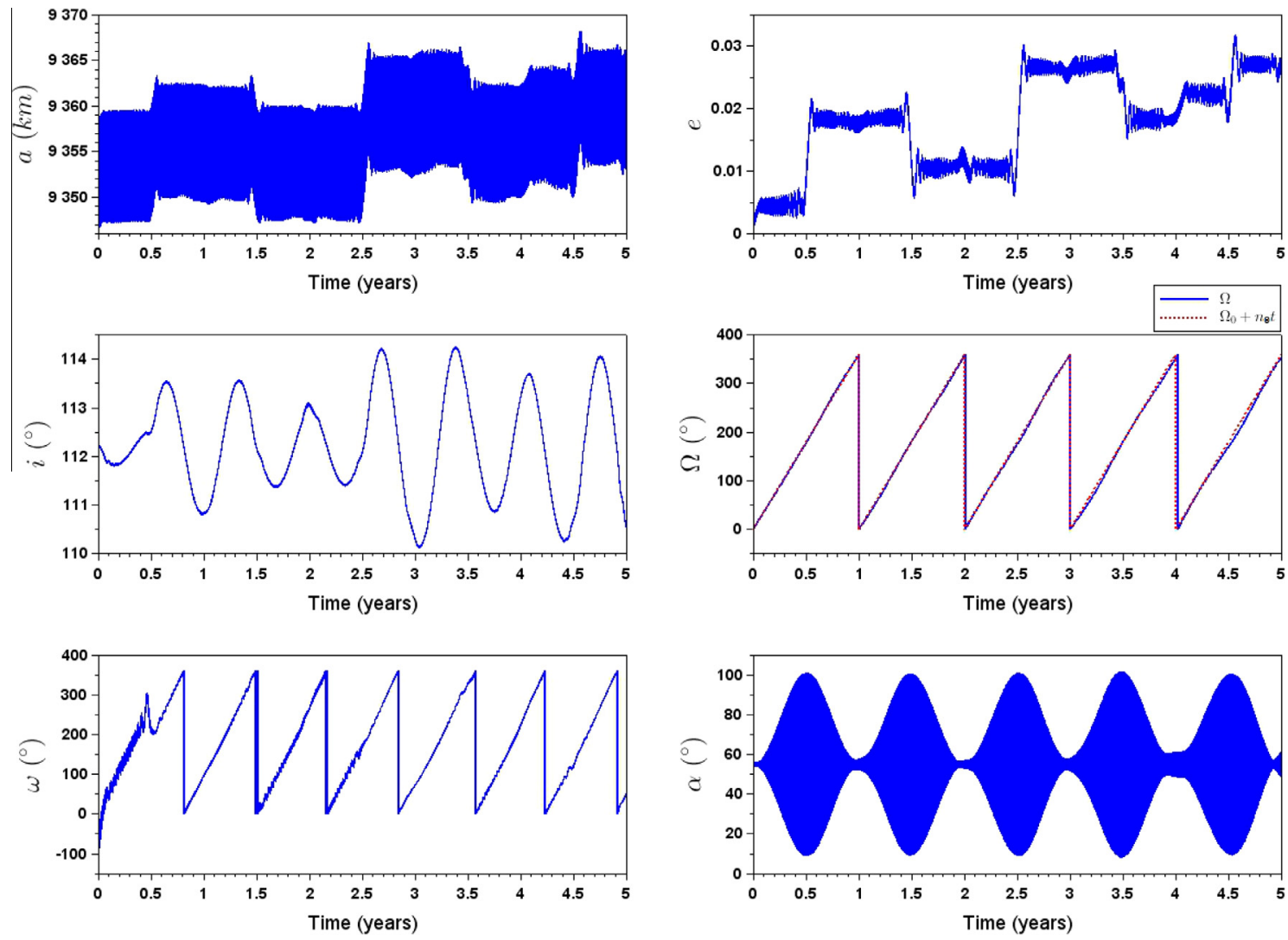

Fig. 4. Classical orbit elements of a Sun-synchronous orbit and Sun angle variation with $\phi=55^{\circ}$ and $\kappa=5.0 \mathrm{~mm} / \mathrm{s}^{2}$ during 5 years.

Table 1

Parameters of the reflector orbit.

\begin{tabular}{lllllll}
\hline$a_{0}$ & $e_{0}$ & $i_{0}$ & $\Omega_{0}$ & $\omega_{0}$ & $f_{0}$ & $\lambda_{\odot 0}$ \\
\hline $9346.6 \mathrm{~km}$ & 0.001 & $112.2^{\circ}$ & $0^{\circ}$ & $-90^{\circ}$ & $0^{\circ}$ & $90^{\circ}$ \\
\hline
\end{tabular}

proposed the development of optically controlled reflector films to control the solar pressure force and hence the deflected shape. Wang et al. (2015) presented an inflatable reflector which support structure is mainly composed of a central hub, a support rib, a support torus, and a tension system. On the other hand, the Sun angle changes periodically as the Earth rotates around the Sun as shown in Figs. 3 and 4, which leads to a variation of the SRPF. Since the inclination is about $112^{\circ}$ during the 5 years of simulation, the oscillation amplitude of the Sun angle increases when the sunlight is not perpendicular to the orbit plane.

One of the disadvantages of the Keplerian Sunsynchronous polar orbits found in this section is the altitude, as shown in Fig. 2. Space debris cannot be ignored for solar reflectors deployed on medium altitude orbits. Additionally, a reflector at altitude $d$ from the Earth's surface will project a spot of diameter of order $D_{s} \sim 0.0093 d$, assuming a perfectly flat reflecting surface (Canady and Allen, 1982). Therefore, for orbiting reflectors placed in a polar orbit with an altitude of $d=3000 \mathrm{~km}$, the spot diameter is approximately $30 \mathrm{~km}$. It would be necessary to deploy a large number of reflectors to increase the total insolation. On the other hand, the Keplerian Sunsynchronous polar orbits obtained in this section show a large variation of the Sun angle as shown in Fig. 3 and Fig. 4. In this light, a family of Earth-centered nonKeplerian orbits will now be discussed in the next section, which permits the deployment of a reflector with a displacement of many times the radius of the Earth. Note that, the distance $d$ must be less than $1.4 \times 10^{6}$ to project a spot of size less than the mean Earth's diameter $(12,742 \mathrm{~km})$.

\section{Earth-centered displaced polar orbits}

The existence of displaced circular two-body orbits has been considered by various authors with applications for solar sails, orbiting reflectors to engineer Mars' climate and multiple mirrors to survive the lunar night (McInnes and Simmons, 1992; Dankowicz, 1994; McInnes, 2004, 2010; Bewick et al., 2011). As shown in Fig. 5, this family of orbits can be generated by orienting the reflector, in such a way, that a component of the SRPF exerted on the reflector is directed normal to the orbit plane (McInnes and Simmons, 1992). The momentum accumulated by the reflector due to SRPF is then offset by the $z$-component of the local gravitational force. In this manner, a family of circular near polar orbits, but displaced behind Earth in the anti-Sun direction, permits a reflector to be deployed at a distance from the Earth's surface, such that, the 

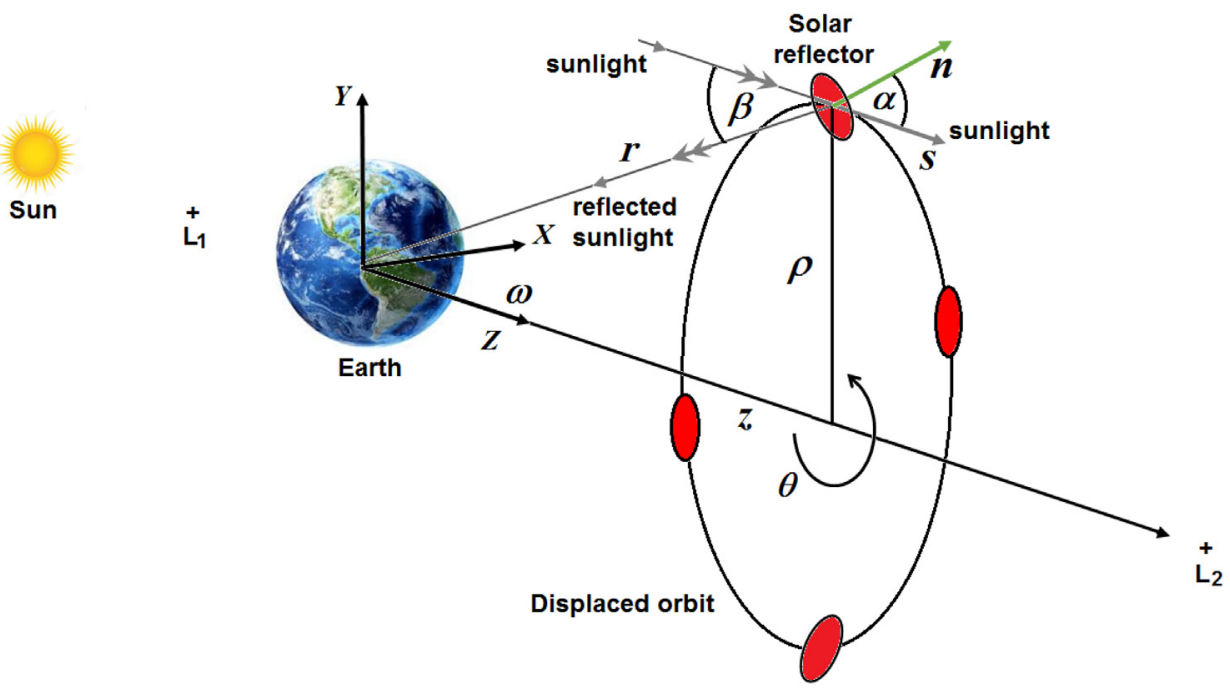

Fig. 5. Solar reflectors on a displaced polar orbit along the Sun-line.

diameter of the spot projected on the surface of the Earth will be of the order of the diameter of the Earth, as demonstrated at the end of this section.

\subsection{Equation of motion}

In order to investigate this family of displaced orbits, a perfectly reflecting space mirror at position $\boldsymbol{r}$ will be considered in a rotating frame of reference, with the origin of the frame at the Earth's center. The axis of rotation $\omega$ will be directed along the Sun-Earth line, and the $Y$-axis of the inertial coordinate system $X Y Z$ is the axis of rotational symmetry, as shown in Fig. 5. Similarly, the reflector normal will be defined by a unit vector $\boldsymbol{n}=[0 \sin \alpha \cos \alpha]^{T}$ fixed in the rotating frame of reference, the characteristic acceleration of the reflector will be denoted by $\kappa$ and the Sun-line will be defined by a unit vector $s=\left[\begin{array}{lll}0 & 0 & 1\end{array}\right]^{T}$. Therefore, with respect to the inertial frame of reference, the reflector must rotate once per orbit due to the reflector attitude being fixed in the rotating frame of reference. In this analysis, it is assumed that the Sun-line is fixed.

The equation of motion of a solar reflector with respect to this rotating frame considering the Earth's gravitational potential, $J_{2}$ effect and uniform radiation field is given by

$\ddot{\boldsymbol{r}}+2 \boldsymbol{\omega} \times \dot{\boldsymbol{r}}+\boldsymbol{\omega} \times(\boldsymbol{\omega} \times \boldsymbol{r})=\boldsymbol{T}-\nabla V$,

where the first and second derivatives of the coordinates are taken with respect to the time, the solar radiation acceleration $\boldsymbol{T}=\kappa \cos ^{2} \alpha \boldsymbol{n}$ and the two-body gravitational potential, including the $J_{2}$ term, is given by

$V=-\frac{\mu}{r}+\mu \frac{J_{2}}{2}\left(\frac{R}{r}\right)^{2} \frac{1}{r}\left(3\left(\frac{y}{r}\right)^{2}-1\right)$.

In the rotating frame of reference, circular displaced orbits require that the first two terms of Eq. (16) must be vanished. Using Earth-centered cylindrical polar coordi- nates $(\rho, \theta, z)$ defined in Fig. 5, the equations of motion for an ideal specular reflector can be written as (McInnes, 2002)

$$
\begin{gathered}
\ddot{\rho}-\rho \dot{\theta}^{2}=-\frac{\mu}{r^{3}} \rho\left(1-\frac{3}{2} J_{2}\left(\frac{R}{r}\right)^{2}\left(\left(5\left(\frac{\rho}{r}\right)^{2}-2\right) \sin ^{2} \theta-1\right)\right) \\
+\kappa \cos ^{2} \alpha \sin \alpha, \\
\rho \ddot{\theta}+2 \dot{\rho} \dot{\theta}=-\frac{3}{2} J_{2} \frac{\mu}{r^{3}} \rho\left(\frac{R}{r}\right)^{2} \sin 2 \theta, \\
\ddot{z}=-\frac{\mu}{r^{3}} z\left(1-\frac{3}{2} J_{2}\left(\frac{R}{r}\right)^{2}\left(5\left(\frac{\rho}{r}\right)^{2} \sin ^{2} \theta-1\right)\right)+\kappa \cos ^{3} \alpha .
\end{gathered}
$$

Since the axis of symmetry $Y$ is parallel to the Earth's polar axis, the left side of the differential Eq. (18) also depends on the cylindrical polar coordinate $\theta$. Thus, we will use $\theta$ as a new independent variable instead of time and perform an average of the resulting equations of motion over one orbit, in order to make the equilibrium conditions as a function of radius $\rho$ and displacement distance $z$.

Substituting the chain rule formula $\dot{\boldsymbol{r}}=\omega(\mathrm{d} \boldsymbol{r} / \mathrm{d} \theta)$, where the orbital angular velocity $\omega=\dot{\theta}$, and $\ddot{\boldsymbol{r}}=\omega(\mathrm{d} \omega / \mathrm{d} \theta)$ $(\mathrm{d} \boldsymbol{r} / \mathrm{d} \theta)+\omega^{2}\left(\mathrm{~d}^{2} \boldsymbol{r} / \mathrm{d} \theta^{2}\right)$ into Eq. (18), and performing the averaging results in the orbit-averaged equation of motions, we have

$$
\begin{aligned}
& \omega^{2} \rho^{\prime \prime}+\omega \omega^{\prime} \rho^{\prime}-\omega^{2} \rho=-\frac{\mu}{r^{3}} \rho\left(1-\frac{3}{4} J_{2}\left(\frac{R}{r}\right)^{2}\left(5\left(\frac{\rho}{r}\right)^{2}-4\right)\right) \\
& \quad+\kappa \cos ^{2} \alpha \sin \alpha \\
& \rho \omega^{\prime}+2 \omega \rho^{\prime}=0 \\
& \omega^{2} z^{\prime \prime}+\omega \omega^{\prime} z^{\prime}=-\frac{\mu}{r^{3}} z\left(1-\frac{3}{4} J_{2}\left(\frac{R}{r}\right)^{2}\left(5\left(\frac{\rho}{r}\right)^{2}-2\right)\right)+\kappa \cos ^{3} \alpha,
\end{aligned}
$$


where the first and second derivatives of the coordinates are taken with respect to $\theta$, i.e., $\rho^{\prime}=\mathrm{d} \rho / \mathrm{d} \theta, \rho^{\prime \prime}=\mathrm{d}^{2} \rho / \mathrm{d} \theta^{2}$ (similar expressions for $\omega$ and $z$ coordinates).

For a circular displaced orbit, it is required that $\rho^{\prime \prime}=z^{\prime \prime}=0$ and $\rho^{\prime}=z^{\prime}=\omega^{\prime}=0$. It can be shown that the required reflector pitch angle $\alpha$ and acceleration $\kappa$ for an equilibrium solution are given by (McInnes, 2002)

$$
\begin{aligned}
& \tan \alpha=\left(\frac{\rho}{z}\right) \frac{1-\left(\frac{\omega}{\omega}\right)^{2}}{1-\frac{3}{2} \frac{\mu}{r^{3}} J_{2}\left(\frac{R}{\omega r}\right)^{2}}, \\
& \kappa=\widetilde{\omega}^{2}\left(1-\frac{3}{2} \frac{\mu}{r^{3}} J_{2}\left(\frac{R}{\widetilde{\omega} r}\right)^{2}\right)\left(1+\tan ^{2} \alpha\right)^{3 / 2} z, \\
& \widetilde{\omega}^{2}=\frac{\mu}{r^{3}}\left(1-\frac{3}{4} J_{2}\left(\frac{R}{r}\right)^{2}\left(5\left(\frac{\rho}{r}\right)^{2}-4\right)\right) .
\end{aligned}
$$

If the space mirror redirects the sunlight towards the Earth, then it is clear from the geometry of the incident ray that the pitch angle $\alpha$ must satisfy the relation $\alpha=\beta / 2$ (see Fig. 5). The required pitch angle is therefore given by McInnes (2002)

$\tan \alpha=\tan \left(\frac{1}{2} \tan ^{-1}\left(\frac{\rho}{z}\right)\right)$.

Therefore, defining this orientation a priori, the required orbital angular velocity of the reflector in order to ensure correct illumination of the Earth by the reflector, and the corresponding reflector acceleration can be obtained from Eq. (20) as

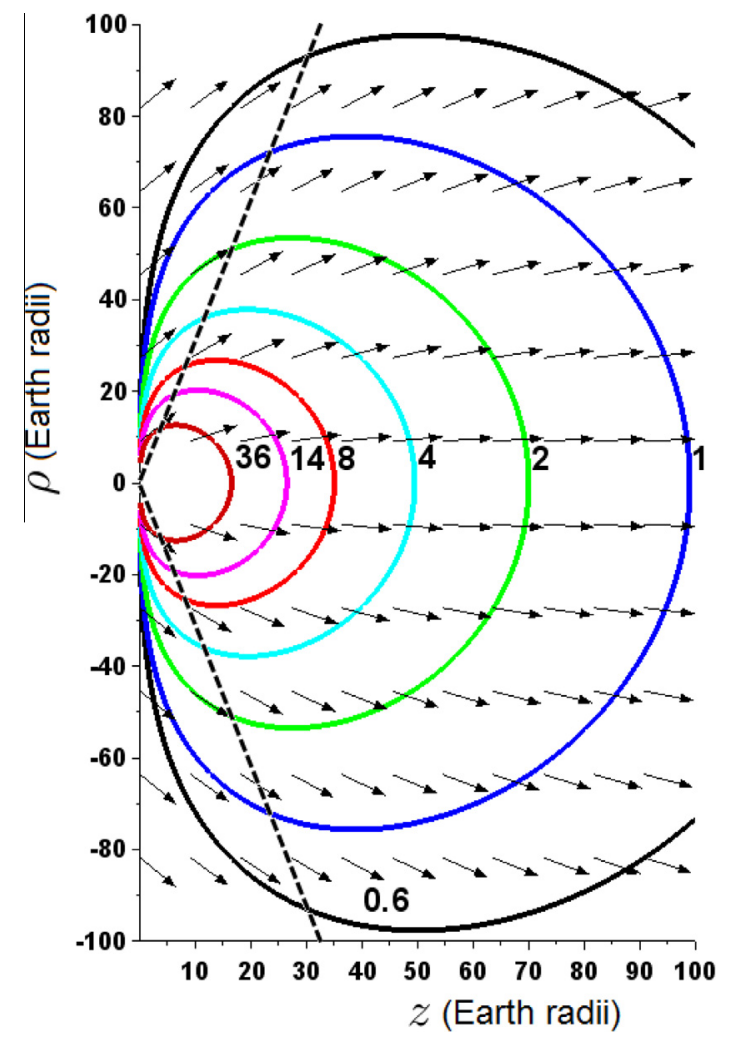

Fig. 6. Reflector requirements for a displaced polar orbit. Contours indicate radiation pressure acceleration $\kappa\left(\mathrm{mms}^{-2}\right)$ and the dashed line indicates the stability boundary (linear stable orbits if $\rho>3.1 z$ ). $\omega=\widetilde{\omega}\left(1-\left(\frac{z}{\rho}\right)\left(1-\frac{3}{2} \frac{\mu}{r^{3}} J_{2}\left(\frac{R}{\widetilde{\omega} r}\right)^{2}\right) \tan \alpha\right)^{1 / 2}$.

From Eq. (20), surfaces of constant reflector acceleration may be generated, with $\kappa$ chosen to be some fixed value. A section of these surfaces is shown in Fig. 6 in the $\rho-z$ plane. It can be seen that the surfaces of constant reflector acceleration have a rotational symmetry about the Sun-line. Additionally, for a given reflector acceleration, orbits with a large radius and small displacement are possible, and therefore, with a pitch angle of order $\alpha \sim 45^{\circ}$. Similarly, orbits with a small radius and large displacement are also possible for the same acceleration, however, with a small pitch angle (i.e. $\alpha \sim 0^{\circ}$ ). Although orbits with large displacement are more desirable due to the projected reflector area being maximized (Canady and Allen, 1982), these orbits are unstable, as will be demonstrated in the next section.

\subsection{Displaced polar orbit stability}

Now that the families of displaced orbits with the appropriate reflector orientation have been established, a linear perturbation analysis will be performed, which in the present case provides necessary conditions for stability and sufficient conditions for instability.

A perturbation $\boldsymbol{\delta}=[\xi \omega \eta]^{T}$ will be added to the reflector position vector at some point $\boldsymbol{r}_{0}=\left[\rho_{0} \omega_{0} z_{0}\right]^{T}$ on the nominal orbit such that $\boldsymbol{r}_{0} \rightarrow \boldsymbol{r}_{0}+\boldsymbol{\delta}$. The radiation pressure acceleration remains constant during the perturbation. Thus, a homogeneous variational equation in the cylindrical components form can be then obtained from the nonlinear equations of motion, Eq. (19) (McInnes, 2004),

$\frac{\mathrm{d}^{2}}{\mathrm{~d} \theta^{2}}\left[\begin{array}{l}\hat{\xi} \\ \hat{\eta}\end{array}\right]+\left[\begin{array}{ll}L_{11} & L_{13} \\ L_{31} & L_{33}\end{array}\right]\left[\begin{array}{l}\hat{\xi} \\ \hat{\eta}\end{array}\right]=\left[\begin{array}{l}0 \\ 0\end{array}\right]$,

where the re-scaled coordinates are defined as

$$
\begin{aligned}
& \hat{\xi}=\xi-\frac{4 \omega^{2} L_{33}}{\left(3 \omega^{2}+L_{11}\right) L_{33}-L_{13} L_{31}} \xi_{0}, \\
& \hat{\eta}=\eta+\frac{4 \omega^{2} L_{31}}{\left(3 \omega^{2}+L_{11}\right) L_{33}-L_{13} L_{31}} \xi_{0}
\end{aligned}
$$

and the linear expansion coefficients of the matrix $\boldsymbol{L}$ are given by

$$
\begin{aligned}
& L_{11}=3+\frac{\mu}{r^{3}}\left(\frac{1}{\omega}\right)^{2}\left(1-3\left(\frac{\rho}{r}\right)^{2}\right)+M_{11}, \\
& L_{13}=-3 \frac{\mu}{r^{3}}\left(\frac{1}{\omega}\right)^{2}\left(\frac{\rho z}{r^{2}}\right)+M_{13}, \\
& L_{31}=-3 \frac{\mu}{r^{3}}\left(\frac{1}{\omega}\right)^{2}\left(\frac{\rho z}{r^{2}}\right)+M_{31}, \\
& L_{33}=\frac{\mu}{r^{3}}\left(\frac{1}{\omega}\right)^{2}\left(1-3\left(\frac{z}{r}\right)^{2}\right)+M_{33}
\end{aligned}
$$


and the coefficients $M_{i j}(i, j=1,3)$ due to Earth's oblateness are of the form

$M_{11}=-\frac{3}{4} \frac{\mu}{r^{3}} J_{2}\left(\frac{R}{\omega r}\right)^{2}\left(\left(1-5\left(\frac{\rho}{r}\right)^{2}\right)\left(5\left(\frac{\rho}{r}\right)^{2}-4\right)+10\left(\frac{\rho z}{r^{2}}\right)^{2}\right)$,

$M_{13}=\frac{15}{4} \frac{\mu}{r^{3}} J_{2}\left(\frac{R}{\omega r}\right)^{2} \frac{\rho z}{r^{2}}\left(7\left(\frac{\rho}{r}\right)^{2}-4\right)$,

$M_{31}=M_{13}$,

$M_{33}=-\frac{3}{4} \frac{\mu}{r^{3}} J_{2}\left(\frac{R}{\omega r}\right)^{2}$.

$\left(\left(1-3\left(\frac{z}{r}\right)^{2}\right)\left(5\left(\frac{\rho}{r}\right)^{2}-2\right)+4\left(\frac{z}{r}\right)^{2}\left(1-5\left(\frac{z}{r}\right)^{2}\right)\right)$.

It should be noted that the orbital angular velocity was ignored in the variational system defined by Eq. (23). This is because all derivatives with respect to $\omega$ in the linear expansion vanish, therefore, the linear system of the variational equation can be reduced to two coupled differential equations (McInnes, 2004). From this perspective, the analysis of linear stability will be determined in the $\rho-z$ plane.

The linear stability of the orbit families can be investigating computing the eigenvalues of the system defined by Eq. (23). In this manner, substituting an exponential solution of the form $\widehat{\boldsymbol{\delta}}=\widehat{\boldsymbol{\delta}}_{0} \exp (\lambda \theta)$, where $\widehat{\boldsymbol{\delta}}=[\hat{\boldsymbol{\xi}} \hat{\eta}]^{T}$, into Eq. (23), the characteristic polynomial of the variational equation is obtained as

$\lambda^{4}+\operatorname{tr}(\boldsymbol{L}) \lambda^{2}+\operatorname{det}(\boldsymbol{L})=0$.

If the trace of the matrix $\operatorname{tr}(\boldsymbol{L})$ and its determinant $\operatorname{det}(\boldsymbol{L})$ are strictly positive, $\lambda$ is purely imaginary, indicating stable, bound oscillations. Examining numerically the stability condition, it is found that $\operatorname{tr}(\boldsymbol{L})$ is strictly positive in the $\rho-z$ plane, while that the contour line for $\operatorname{det}(\boldsymbol{L})=0$ is represented by the curve $\rho=3.1 z$, such that, $\operatorname{det}(\boldsymbol{L})$ is positive in the region $\rho>3.1 z$. Thus, the boundary $\rho=3.1 z$, denoted by the dashed line in Fig. 6, divides into stable and unstable the regions in the $\rho-z$ plane, with stable orbits in the region $\rho>3.1 z$.

Fig. 7 shows two 15 day Earth-centered orbits, as well as the variation of the pitch angle for each orbit, obtained by a numerical integration of the full-nonlinear equations of motion, Eq. (18). The first plot in the first two rows shows the $(x, y)$ projection, and the second one contains the $(x, z)$ projection. Again, the numerical integration is performed through the Bulirsch-Stoer algorithm. Orbit I has an initial radius $\rho_{0}=10.0$ Earth radii and an initial displacement $z_{0}=0.25$ Earth radii with a perturbation $\delta=10^{-3} \boldsymbol{r}_{0}$, as can be seen in Fig. 7(a)-(b). Although the linear analysis is not sufficient to conclude the stability of orbits with large radius and small displacement, such that $\rho_{0}>3.1 z_{0}$, the numerical experiment suggests that orbits with those characteristics are in fact stable, i.e., orbits with a relatively modest displacement are still able to cancel the momentum accumulated by the reflector due to solar radiation perturbation by the $z$-component of the Earth gravitational force, with the orbit plane remaining normal to the Sun-Earth line during the 15 day propagation, as shown in Fig. 7(c) $\left(\alpha \sim 45^{\circ}\right)$.

Finally, it can be seen that orbit II (Figs. 7(d)-(e)) represents a typical unbound orbit, which initial radius $\rho_{0}=2.0$ Earth radii, initial displacement $z_{0}=10.0$ Earth radii and similar perturbation. Clearly, a large displacement leads to the SRPF driving the reflector out of the Earth's gravitational potential. On the other hand, comparing Fig. 7(c) and (f), it can be noted that a large displacement reduces the pitch angle, and therefore, the energy radiated from a space reflector (Eq. (3)), placed on non-Keplerian orbits with a $z$-component much larger than its radius, will be increased. From this analysis, unstable orbits could then be more favorable for deploying solar reflectors than uncontrolled bound solutions. Although unstable, previous studies have shown the family of non-Keplerian unstable orbits are strictly controllable (McInnes, 2004). Thus, if unstable orbits are in principle controllable, a control scheme will ensure that the reflector pitch angle will approach zero as shown in Eq. (21). Additionally, note that a controlled orbit with initial radius $\rho_{0}=2.0$ Earth radii and initial displacement $z_{0}=10.0$ Earth radii, will project a spot of diameter of order $D_{s} \sim 545 \mathrm{~km}$, practically twenty times the diameter of the spot projected by the Keplerian polar orbits found in this study.

\section{Solar reflector fabrication}

The mass required to fabricate a solar reflector utilizing the polar orbits discussed in Sections 3 and 4 will now be considered. Using Eq. (4), it can be shown that for an increment of $0.5 \mathrm{~K}$ in the Earth's mean temperature, it would be necessary to increase solar insolation of $1.7 \mathrm{~W} \mathrm{~m}^{-2}$, i.e., $\delta I / I=0.5 \%$. For a characteristics acceleration of $1.0 \mathrm{~mm} \mathrm{~s}^{-2}$ and $5.0 \mathrm{~mm} \mathrm{~s}^{-2}$ (values used in the Keplerian polar orbits obtained in Section 3), corresponds an area density of order $9.0 \mathrm{gm}^{-2}$ and $1.8 \mathrm{gm}^{-2}$, respectively. These values are similar for high performance square solar sails (McInnes, 2004).

Considering the Sun-synchronous polar orbits shown in Figs. 3 and 4, it is found that the average reflector angle $\alpha$ for both orbits is of order $57^{\circ}$. On the other hand, non-Keplerian orbits with a radius $\rho$ of 18 Earth radii and 11 Earth radii, with a small displacement $z$ of 0.25 Earth radii, show an acceleration of $1.0 \mathrm{~mm} \mathrm{~s}^{-2}$ and $5.0 \mathrm{~mm} \mathrm{~s}^{-2}$, respectively. Using Eq. (21), the reflector angle can also be obtained and it is found of order $44^{\circ}$ in both cases. Similarly, displaced orbits with a radius $\rho$ of 2 Earth radii with large displacements $z$ of 98 Earth radii and 44 Earth radii, show an acceleration of $1.0 \mathrm{~mm} \mathrm{~s}^{-2}$ and $5.0 \mathrm{~mm} \mathrm{~s}^{-2}$, respectively, and a reflector angle of order $1^{\circ}$. The required reflector area to obtain an increase $\delta I$ of $1.7 \mathrm{~W} \mathrm{~m}^{-2}$ in solar insolation $I$ can therefore be obtained from Eq. (4) as 
(a) $x-y$

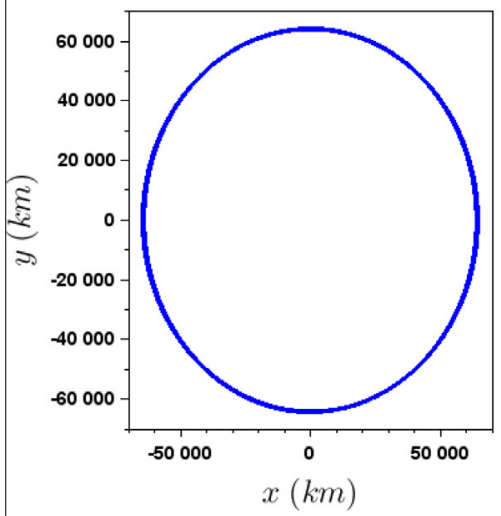

(d) $x-y$

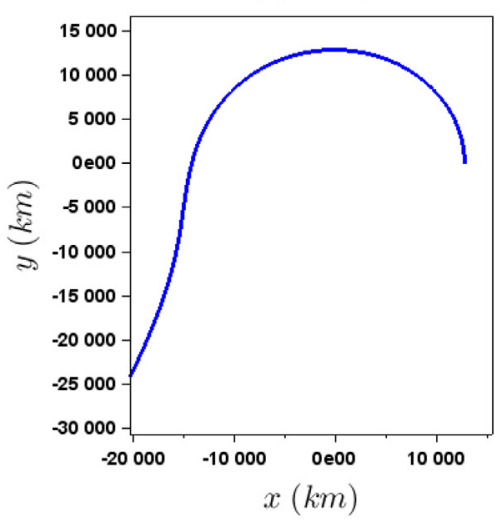

(b) $x-z$

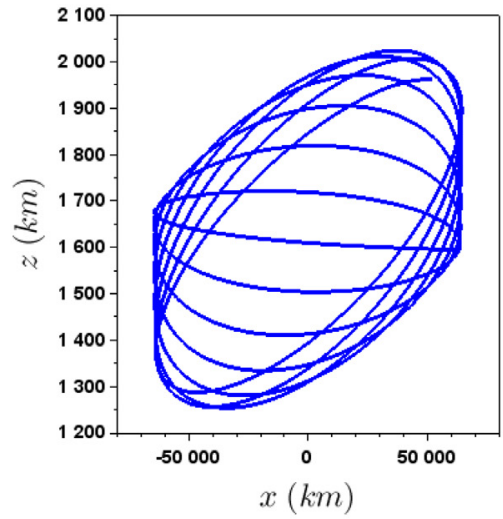

(e) $x-z$

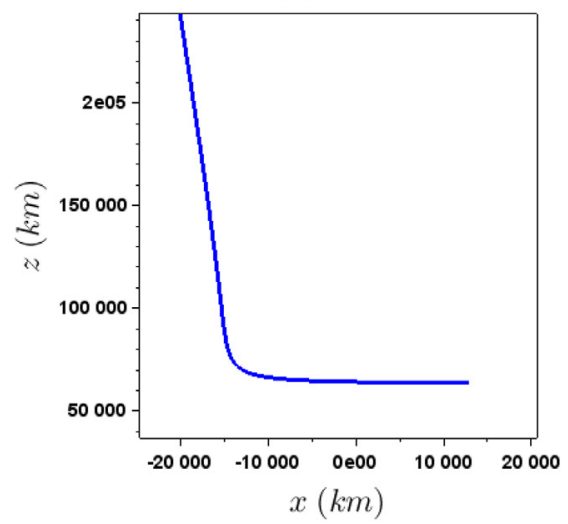

(c)

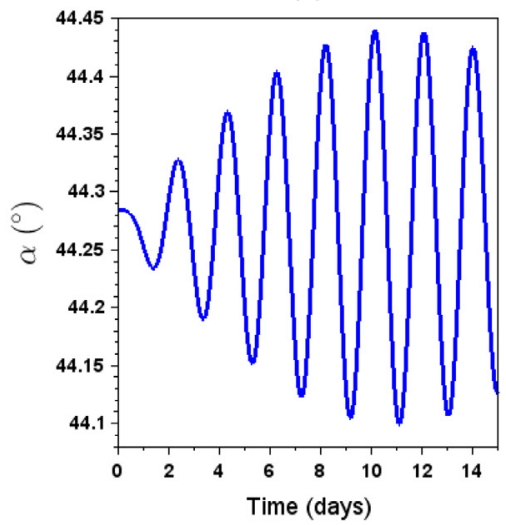

(f)

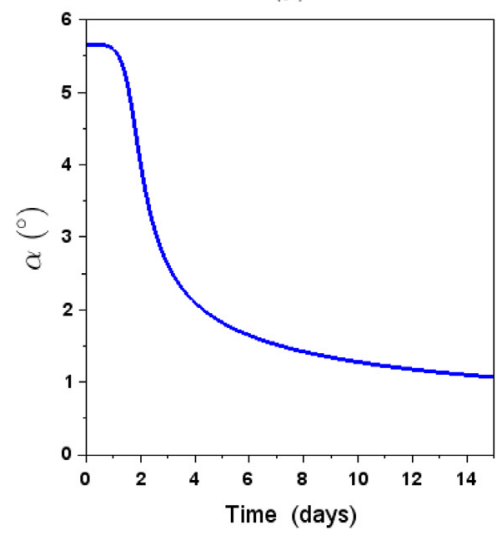

Fig. 7. (a) and (b) Uncontrolled stable orbit I ( $\rho_{0}=10.0$ Earth radii, $z_{0}=0.25$ Earth radii, $\left.\xi=10^{-3} \rho_{0}, \eta_{0}=10^{-3} z_{0}\right)$, (d) and (e) Unstable orbit II ( $\rho_{0}=2.0$ Earth radii, $z_{0}=10.0$ Earth radii, $\xi=10^{-3} \rho_{0}, \eta_{0}=10^{-3} z_{0}$ ). Variation of the pitch angle $\alpha$ for orbits I (c) and II (f). First plot: $(x, y)$ projection; Second plot: $(x, z)$ projection.

$A=\frac{4 \pi R^{2}}{F \cos \alpha} \delta I$

The reflector total mass $m$ is then equal to $\sigma_{A} A$.

Table 2 shows the mass and area of the reflector deployed in the orbits considered for characteristic accelerations of $1.0 \mathrm{~mm} \mathrm{~s}^{-2}$ and $5.0 \mathrm{~mm} \mathrm{~s}^{-2}$, the mean reflector pitch angle and the diameter of mirror (denoted by $D_{r}$ ). As can be seen in Table 2, the use of unstable displaced orbits could reduce the mass requirements and the size of the reflector. Note that a mirror diameter of order $500-700 \mathrm{~km}$ would envisage many smaller reflectors distributed about the same orbit (Early, 1989; Angel, 2006; Pearson et al., 2006).
On the other hand, the fabrication and launching of a reflector from the Earth with a total mass of order $10^{9} \mathrm{~kg}$ introduces engineering challenges. For comparison, the total mass of the Great pyramid of Giza is of order $10^{8} \mathrm{~kg}$ (McInnes, 2006). In this light, Angel (2006) proposed using electromagnetic launch followed by ion propulsion to transport a total payload mass of $1.8 \times 10^{10} \mathrm{~kg}$ from the Earth to the Earth-Sun $\mathrm{L}_{1}$ Lagrange point. However, an advanced industrial space capacity with the ability to exploit extraterrestrial resources would be able to avoid the launching of the reflector from the Earth's surface to the polar orbit. Previous studies suggest that the mass requirement for the fabrication of a reflector

Table 2

Reflector physical properties.

\begin{tabular}{lllll}
\hline Orbit type & $\kappa\left(\mathrm{mms}^{-2}\right)$ & $\alpha\left(^{\circ}\right)$ & $A_{r}\left(\mathrm{~km}^{2}\right)$ & $m_{r}(\mathrm{~kg})$ \\
\hline Sun-synchronous orbit (Fig. 3) & 1.0 & 57 & $1.1 \times 10^{6}$ & $1.1 \times 10^{10}$ \\
Sun-synchronous orbit (Fig. 4) & 5.0 & 57 & $1.1 \times 10^{6}$ & $2.1 \times 10^{9}$ \\
Stable displaced orbit $\left(\rho=18 R^{*}, z=0.25 R\right)$ & 1.0 & 44 & $8.7 \times 10^{5}$ & $7.8 \times 10^{9}$ \\
Stable displaced orbit $(\rho=11 R, z=0.25 R)$ & 5.0 & 44 & $8.7 \times 10^{5}$ & 680 \\
Unstable displaced orbit $(\rho=98 R, z=2 R)$ & 1.0 & 0.6 & $6.2 \times 10^{5}$ & 590 \\
Unstable displaced orbit $(\rho=44 R, z=2 R)$ & 5.0 & 1.3 & $6.2 \times 10^{9}$ & $5.6 \times 10^{9}$ \\
\hline
\end{tabular}

${ }^{*} R=6371 \mathrm{~km}$ (Earth radius). 
could be satisfied by M-type asteroids, which are relatively abundant in Nickel-Iron materials (Gehrels, 1979; Sanchez and McInnes, 2010, 2011; Sanchez et al., 2012). Assuming that an asteroid has a bulk density of $7860 \mathrm{~kg} \mathrm{~m}^{-3}$ (iron density), and a reflector density of $1.8 \mathrm{gm}^{-2}$, the material could be extracted from the surface of the asteroid using solar concentrators, mass driver equipped landers or by spin fragmentation (Melosh et al., 1994; Harris, 1996; Olds et al., 2004) and used to manufacture a thin metallic film of order $0.1 \mu \mathrm{m}$ from a M-type asteroid with a radius of $70 \mathrm{~m}$ (Lippmann, 1972). This thickness is clearly challenging, however terrestrial experiments, performed only a laboratory scale, have been produced thin metallic film of comparable thickness (Drexler, 1979).

\section{Conclusions}

Large-scale geo-engineering proposals for Earth climate warming using orbiting solar reflectors have been considered in this paper. Firstly, Sun-synchronous frozen polar orbits have been presented which are suitable for use by solar reflectors placed on Keplerian orbits. By considering the two-body problem, including $J_{2}$ perturbation and solar radiation pressure, initial parameters for Sun-synchronous frozen orbits have been obtained. Numerical simulations of near circular orbits showed that, although orbital elements such as semi-major axis, eccentricity and inclination remain practically close to the desired values, the variation of the argument of perigee means that the orbit cannot keep the Sun-synchronous frozen conditions for a long time with a fixed attitude. Therefore, an attitude control scheme should be employed to achieve the Sun-synchronous condition.

In order to avoid the instability presented in Keplerian orbits, a family of displaced Earth orbits has been investigated. Again, considering the Earth's gravity and the $J_{2}$ effect, the required angular velocity of equilibrium orbits with respect to a rotating frame and the reflector acceleration have been derived as a function of the radius and displacement of the orbit. It has been show that, with the same characteristic acceleration, it is possible to obtain a non-Keplerian orbit with large radius and small displacement or small radius and large displacement. However, the linear stability analysis and numerical simulations have shown that only orbits relatively close to the planetary terminator are stable, while orbits with a large displacement are unstable. Thus, displaced orbits close to the planetary terminator would be clearly more advantageous for their use in large-scale climate engineering than Earth-centered orbits due to their natural stability and that the pitch angle would remain about $45^{\circ}$, i.e., a control scheme to maintain the stability and large variations of the Sun angle would be avoided.

The large scale of the space-based solar reflector makes it impossible to assemble and lunched from the Earth. In this manner, exploiting extra-terrestrial resources as M-type asteroids, has been proposed by several studies for fabrication of such space mirrors.
Finally, although the non-spherical perturbation is dominated by the $J_{2}$ term for low altitude orbits, the inclusion of $J_{2}$ effects in the orbital dynamics increases the accuracy of the model. Additionally, the analytical expressions of the required angular velocity and reflector acceleration for displaced orbits, obtained in this study, can be used in any two-body system that includes $J_{2}$ perturbation (e.g. Sun-Mars system). Atmospheric drag, a rotating Sun-line and third-body gravitational perturbations may be included, in such a way that a more realistic description of the most suitable orbits for solar reflectors could be obtained for Earth climate warming.

\section{Acknowledgments}

Firstly, the authors thank the financial support of the FAPESP (São Paulo Research Foundation, Brazil), Grants 2011/08171-3, 2013/03233-6, 2015/00559-3 and the CNPq (National Council for Scientific and Technological Development, Brazil), and the technical support of University of Glasgow. C.R.M was support by a Leverhlume Trust Fellowship (2014-2015). Finally, the authors are also grateful to Dr. J. Simo of the School of Engineering at University of Central Lancashire from United Kingdom, for ideas and suggestions, particularly in the geocentric Sun-synchronous frozen orbits.

\section{References}

Allen, R.G., Trezza, R., Tasumi, M., 2006. Analytical integrated functions for daily solar radiation on slopes. Agric. For. Meteorol. 139 (1-2), 5573. http://dx.doi.org/10.1016/j.agrformet.2006.05.012.

Angel, R., 2006. Feasibility of cooling the Earth with a cloud of small spacecraft near the inner Lagrange point (L1). P. Natl. Acad. Sci. USA 103 (46), 17184-17189. http://dx.doi.org/10.1073/pnas.0608163103.

Angell, J.K., 1988. Impact of El Niño on the delineation of tropospheric cooling due to volcanic eruptions. J. Geophys. Res. (Atmos.) 93 (D4), 3697-3704. http://dx.doi.org/10.1029/JD093iD04p03697.

Angell, J.K., Korshover, J., 1984. Comparison of tropospheric temperatures following Agung and El Chichón volcanic eruptions. Mon. Wea. Rev. 112 (7), 1457-1463. http://dx.doi.org/10.1175/1520-0493 $(1984)<1121457:$ COTTFA>2.0.CO;2.

Bate, R.R., Mueller, D.D., White, J.E., 1971. Fundamentals of Astrodynamics. Dover, New York.

Battin, R.H., 1987. An Introduction to the Mathematics and Methods of Astrodynamics. AIAA Education Series, New York.

Berglund, N., Gentz, B., 2001. Metastability in simple climate models: pathwise analysis of slowly driven Langevin equations. In: Proc. of the 2nd Workshop on Stochastic Climate Models, Chorin, Germany.

Bewick, R., Sanchez, J.P., McInnes, C.R., 2011. Use of orbiting reflectors to decrease the technological challenges of surviving the lunar night. In: 62nd International Astronautical Congress 2011, Oct. 03-07, Cape Town. Paper ID 10127.

Bewick, R., Sanchez, J.P., McInnes, C.R., 2012. The feasibility of using an L1 positioned dust cloud as a method of space-based geoengineering. Adv. Space Res. 49 (7), 1212-1228. http://dx.doi.org/10.1016/j. asr.2012.01.010.

Bewick, R., Lücking, C., Colombo, C., et al., 2013. Heliotropic dust rings for Earth climate engineering. Adv. Space Res. 51 (7), 1121-1144. http://dx.doi.org/10.1016/j.asr.2012.10.024.

Borggräfe, A., Heiligers, J., Ceriotti, M., et al., 2014. Inverse problem for shape control of flexible space reflectors using distributed solar 
pressure. Smart Mater. Struct. 23 (7), 075026. http://dx.doi.org/ 10.1088/0964-1726/23/7/075026.

Borggräfe, A., Heiligers, J., Ceriotti, M., et al., 2015. Shape control of slack space reflectors using modulated solar pressure. Proc. R. Soc. A 471 (2179), 20150119. http://dx.doi.org/10.1098/rspa.2015.0119.

Canady, J.E., Jr., Allen, J.L., Jr., 1982. Illumination From Space With Orbiting Solar-Reflector Spacecraft, NASA TP-2065.

Coffey, S.L., Deprit, A., Deprit, E., 1994. Frozen orbits for satellites close to an Earth-like planet. Celest. Mech. Dyn. Astron. 59 (1), 37-72. http://dx.doi.org/10.1007/BF00691970.

Dankowicz, H., 1994. Some special orbits in the two-body problem with radiation pressure. Celest. Mech. Dyn. Astron. 58 (4), 353-370. http:// dx.doi.org/10.1007/BF00692010.

Drexler, K.E., 1979. High performance solar sails and related reflecting devices. In: 4th Princeton/AIAA Conference on Space Manufacturing Facilities, Princeton, Paper AIAA-79- 1418.

Early, J.T., 1989. Space-based solar shield to offset greenhouse effect. J. Br. Interplanet. Soc. 42, 567-569.

Ehricke, K.A., 1979. Space light: space industrial enhancement of the solar option. Acta Astronaut. 6 (12), 1515-1633.

Emanuel, K.A., 2002. Simple model of multiple climate regimes. J. Geophys. Res. (Atmos.) 107 (D9). http://dx.doi.org/10.1029/ 2001JD001002. ACL 4-1, CiteID 4077.

Free, M., Robock, A., 1999. Global warming in the context of the little ice age. J. Geophys. Res. 104 (D16), 19057-19070.

Fogg, M.J., 1995. Terraforming: engineering planetary environments. SAE Int.

Gehrels, T., 1979. Asteroids. University of Arizona Press, Tucson.

Glaser, P.E., 1968. Power from the sun: its future. Science 162, 857-861.

Gong, S., Li, J., Baoyin, H., et al., 2012. A new solar sail orbit. Sci. Chin. Technol. Sci. 55 (3), 848-855. http://dx.doi.org/10.1007/s11431-0114691-7.

Hansen, J., Lacis, A., Ruedy, R., et al., 1992. Potential climate impact of Mount Pinatubo eruption. Geophys. Res. Lett. 19 (2), 215-218. http:// dx.doi.org/10.1029/91GL02788.

Harris, A.W., 1996. The rotation rates of very small asteroids: evidence for 'Rubble Pile' structure. Lunar Planet. Sci. 27.

Hedgepeth, J.M., Miller, R.K., Knapp, K., 1981. Conceptual design studies for large free-flying solar-reflector spacecraft. NASA Contractor Report 3438.

Hudson, H.S., 1991. A space parasol as a countermeasure against the greenhouse effect. J. Br. Interplanet. Soc. 44, 139-144.

Le Roy Ladurie, E., 1971. Times of Feast, Times of Famine: A History of Climate Since the Year 1000. Doubleday, Garden City, NY, Translated from the French by Barbara Bray.

Leary, W.E., 1993. Russians to test space mirror as giant night light for Earth-New York Times. The New York Times-Breaking News, World News \& Multimedia, The New York Times, 12 Jan. 1993. Web. 11 Dec. 2011. <http://www.nytimes.com/1993/01/12/science/russians-totest-space-mirror-as-giant-night-light-for-earth.html?pagewanted= all $>$ (accessed 17.10.2015)

Lippmann, M.E., 1972. In-space fabrication of thin-film structures. NASA.

Maunter, M., Parks, K., 1990. Space-based climate control, engineering, construction and operations in space II. In: Proc. Space. American Society of Civil Engineers.

McGuffie, K., Henderson-Sellers, A., 2005. A Climate Modelling Primer, third ed. Wiley.

McInnes, C.R., 2002. Non-keplerian orbits for mars solar reflectors. J. Br. Interplanet. Soc. 55, 74-84.

McInnes, C.R., 2002. Minimum mass solar shield for terrestrial climate control. J. Br. Interplanet. Soc. 55, 307-311.

McInnes, C., 2004. Solar Sailing: Technology, Dynamics and Mission Applications. Springer Praxis Books.
McInnes, C.R., 2006. Planetary macro-engineering using orbiting solar reflectors. In: Macro-engineering: A Challenge for the Future. Springer, Berlin, pp. 215-250.

McInnes, C.R., 2010. Space-based geoengineering: challenges and requirements. Proc. Inst. Mech. Eng. C J. Mech. Eng. Sci. 224 (3), 571-580. http://dx.doi.org/10.1243/09544062JMES1439.

McInnes, C.R., 2010. Mars Climate Engineering using Orbiting Solar Reflectors. Mars: Prospective Energy and Material Resources. Springer-Verlag.

McInnes, C.R., Simmons, J.F.L., 1992. Halo orbits for solar sails II geocentric case. J. Spacecraft Rockets 29 (4), 472-479. http://dx.doi. org $/ 10.2514 / 3.55639$.

Melosh, H.J., Nemchinov, I.V., Zetzer, Y.I., 1994. Non-nuclear strategies for deflecting comets and asteroids. In: Gehrels, T. (Ed.), Hazards Due to Comets and Asteroids. University of Arizona Press.

Muller, R.A., MacDonald, G.J., 1997. Glacial cycles and astronomical forcing. Science 277, 215-218.

Oberg, J.E., 1981. New Earths: Restructuring Earth and Other Planets. New American Library Inc, New York.

Oberth, H., 1972. Ways to spaceflight, NASA Technical Translation, TT F-622.

Olds, J., Charania, A., Graham, M., et al., 2004. The league of extraordinary machines: a rapid and scalable approach to planetary defense against asteroid impactors. NASA Institute for Advanced Concepts.

Pearson, J., Oldson, J., Levin, E., 2006. Earth rings for planetary environment control. Acta Astronaut. 58 (1), 44-57. http://dx.doi.org/ 10.1016/j.actaastro.2005.03.071.

Press, W.H., Teukolsky, S.A., Vetterling, W.T., et al., 1992. Numerical Recipes in C: The Art of Scientic Computing, second ed. Cambridge University Press, Cambridge.

Sanchez, J.P., McInnes, C., 2010. Accessibility of the resources of near Earth space using multi-impulse transfers. In: AIAA/AAS Astrodynamics Specialist Conference, Toronto, Canada. $<$ http://strathprints. strath.ac.uk/26354/>.

Sanchez, J.P., McInnes, C., 2011. Asteroid resource map for near-earth space. J. Spacecraft Rockets 48 (1), 153-165. http://dx.doi.org/ $10.2514 / 1.49851$

Sanchez, J.P., Garcia Yarnoz, D., Alessi, E.M., et al., 2012. Gravitational capture opportunities for asteroid retrieval missions. In: 63rd International Astronautical Congress, International Astronautical Federation, Naples. $<$ http://strathprints.strath.ac.uk/41127/>.

Stoer, J., Bulirsch, R., 1980. Introduction to Numerical Analysis, 1st ed. Springer Verlag, New York.

Teller, E., Hyde, R., Ishikawa, M., et al., 2004. Active climate stabilization: presentlyfeasible albedo-control approaches to prevention of both types of climate change. In: Symposium on Macro-Engineering Options for Climate Change Management and Mitigation, CambridgeMIT Institute, Cambridge.

Tscherbakova, N.N., Beletskii, V.V., Sazonov, V.V., 1999. Stabilization of heliosynchronous orbits of an Earth's artificial satellite by solar pressure. Cosmic Res. 37 (4), 393-403.

Wang, C.G., Xia, Z.M., Tan, H.F., 2015. Initial shape design and stability analysis of rib for inflatable deployable reflector. AIAA J. 53 (2), 486492. http://dx.doi.org/10.2514/1.J053469.

Zielinski, G.A., Mayewski, P.A., Meeker, L.D., et al., 1996. Potential atmospheric impact of the Toba Mega-Eruption $\sim 71,000$ years ago. Geophys. Res. Lett. 23 (8), 837-840. http://dx.doi.org/10.1029/ 96GL00706.

Zuev, V.V., Zueva, N.E., Saveljeva, E.S., 2015. Temperature and ozone anomalies as indicators of volcanic soot in the stratosphere. Atmos. Ocean. Opt. 28 (1), 100-106. http://dx.doi.org/10.1134/S10248560150 10169. 